\title{
Middle Ear Virtual Endoscopy in Malleus Fracture and Dislocation
}

\author{
Ophir Ilan ${ }^{*}$, , Phillip Berman ${ }^{\mathrm{b}}$, Jacob Sosna ${ }^{\mathrm{b}}$ and Menachem Gross ${ }^{\mathrm{a}}$
}

\author{
Department of Otolaryngology, ${ }^{a}$ Head \& Neck Surgery and ${ }^{b}$ Radiology, Hadassah Hebrew-University Medical Center, \\ Jerusalem, Israel
}

\begin{abstract}
Ossicular injury is a frequent complication of head trauma and temporal bone fractures. An isolated fracture of the malleus with lateral dislocation of the manubrium is extremely rare. High-resolution computed tomography is the method of choice for evaluation of ossicular trauma. Virtual endoscopy (VE) is a novel technique that provides a realistic surface rendering of various organs that can be applied for the use of three-dimensional (3D) reconstruction. We report a case of malleus handle fracture and dislocation after head trauma with confirmation of the diagnosis by VE of the middle ear.
\end{abstract}

Keywords: Malleus, dislocation, fracture, computed tomography, virtual endoscopy.

\section{INTRODUCTION}

Malleus dislocation and fracture is an infrequently diagnosed and reported condition associated with head trauma and skull base fracture. Although less then 50 cases have been reported in the literature in recent years, it is suggested that the actual frequency of malleus fracture is higher and that this condition is under-diagnosed [1]. Malleus fractures can be caused by head trauma, direct injury to the tympanic membrane or barotrauma. Clinical findings are: abnormal mobility of the manubrium mallei during the Valsalva maneuvre or pneumomassage of the ear drum on otoscopy, conductive hearing loss, and abnormal high compliance tympanometry (AD).

High resolution computed tomography (CT) of the temporal bone is the method of choice for the evaluation of ossicular chain integrity [2]. Axial high resolution CT scans can ordinarily demonstrate the exact nature and placement of the ossicular fractures and dislocations [3]. Recently, virtual endoscopy (VE) of the middle ear was used to assess middle ear pathologies and ossicular injuries perioperatively by obtaining three-dimensional (3D) reconstructions of the tympanic cavity using the standard temporal bone CT scan as a template [4-6]. This method adds information not obtained by the standard temporal bone CT scan.

In this report, we describe a unique clinical case of malleus fracture and dislocation using VE with discussion on the application of this technique in middle ear pathologies.

\section{CASE REPORT}

A 23-year-old man presented to our out-patient clinic with right side hearing loss that developed after severe head trauma associated with a right temporal bone fracture. Physical examination of the right ear by microscope revealed an intact tympanic membrane covering a fractured and laterally dislocated handle of the malleus. The fractured segment was displaced and attached to the anterior aspect of the external auditory canal. Pure tone audiometry revealed right severe

*Address correspondence to this author at the Department of Otolaryngology, Head \& Neck Surgery, Hadassah Hebrew-University Medical Center, Jerusalem, Israel; E-mail: ofirilan@gmail.com mixed hearing loss with 50dB air-bone gap. High resolution CT-scan of the temporal bone was performed on the Phillips MX8000-Q scanner, Phillips Medical Systems. Scanning parameters were: slice width - $0.6 \mathrm{~mm}$, slice increment $0.3 \mathrm{~mm}$, pitch $-0.625 \mathrm{~mm}, 180 \mathrm{~mA}$. CT of the temporal bone revealed longitudinal and transverse fractures in the right temporal bone continuing to the middle ear and crossing the tegmen tympani with lateral dislocation of the malleus. The scan demonstrated fracture and dislocation of the handle of the malleus. The incus and head of malleus were not demonstrated in their proper position in the middle ear cavity (Fig. 1). A $3 \mathrm{D}$ reconstruction was obtained using the axial $\mathrm{CT}$ as data to form a VE view of the right and left middle ears using the Phillips Extended Brilliance Workspace 2.0.1. Images were reconstructed in the axial plane at $0.6 \mathrm{~mm}$ intervals using a bone reconstruction algorithm.

Fig. (2) demonstrated an otoscopic virtual view of the normal left ear as compared to the same view obtained fom the right ear. The left ear presented normal anatomy and relations of the ossicular chain and the external auditory canal (Fig. 2a). However, VE of the right ear showed the fractured and laterally and anteriorly dislocated handle of the malleus at horizontal position. This segment is disconnected from the malleus head which is hidden by a bony ridge, formed by the temporal bone fracture (Fig. 2b).

The ossicular chain is not seen in the middle ear cavity. Additional information regarding the ossicular chain can be obtained from VE by rotating the view to angles impossible by normal otoscopy.

Fig. (3), displays a rotated view from the promontorium to the external ear canal. This view reveals the epitympanic location of the malleus head and incus, previously hidden by the ridge of fractured temporal bone.

\section{DISCUSSION}

Ossicular chain fractures and discontinuity after head trauma are uncommon middle ear pathologies, presented by conductive hearing loss, and can be easily missed. In recent years, high resolution CT scan became the modality of choice in the evaluation of middle ear structures. Most of middle ear and ossicular chain pathologies can be assessed 


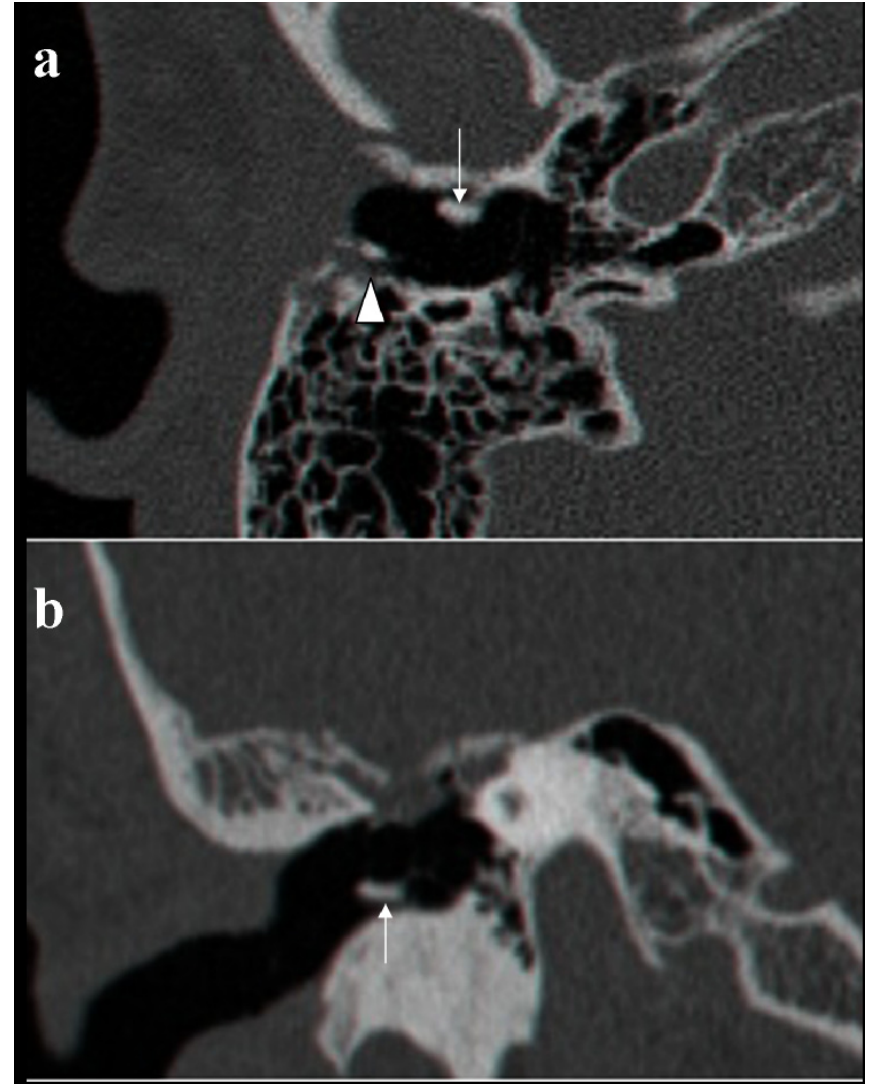

Fig. (1). (a) Axial bone window high resolution CT-scan of the right ear demonstrates the fractured and dislocated handle of malleus displaced laterally (white arrow) and longitudinal temporal bone fracture line (arrowhead). (b) Coronal CT-scan demonstrates lateral displacement of the fractured handle of the malleus (arrow). Tegmen tympani defect with soft tissue opacification of the epitympanicum suspected for meningoencephalocele.

through 2D CT scan. In the last decade, with the advancement of computer technology and the availability of high performance processors, 3D reconstruction and VE of the middle ear cavity became achievable. Martin et al. have previously demonstrated the advantage of VE over 2D spiral CT in the diagnosis of dislocation of the ossicles, and added value of this imaging modality in the preoperative planning of middle ear surgery [5].

In the present case, an uncommon malleus handle fracture with laterally dislocated manubrium is visualized by high resolution CT of the right ear. The position of the head of the malleus was abnormal. VE reconstruction of the right ear verified the $2 \mathrm{D}$ scan finding and added important information. The head of the malleus and incus were positioned behind a bony ridge, formed by the temporal bone fracture. This data is important for surgical preoperative planning. Unfortunately, the stapes cannot be visualized in this method since it is too small to be picked up at the normal slice width. Several surgical approches have been described in the literature for the reduction and fixation of isolated malleus handle fracture [7]. These approches include raising a tympanomeatal flap exposing the malleus and incus [7]. A recent publication describes splinting the broken manubrium to the lenticular process of the incus by a preformed piece of bone [7]. In the present case, raising a tympanomeatal flap would

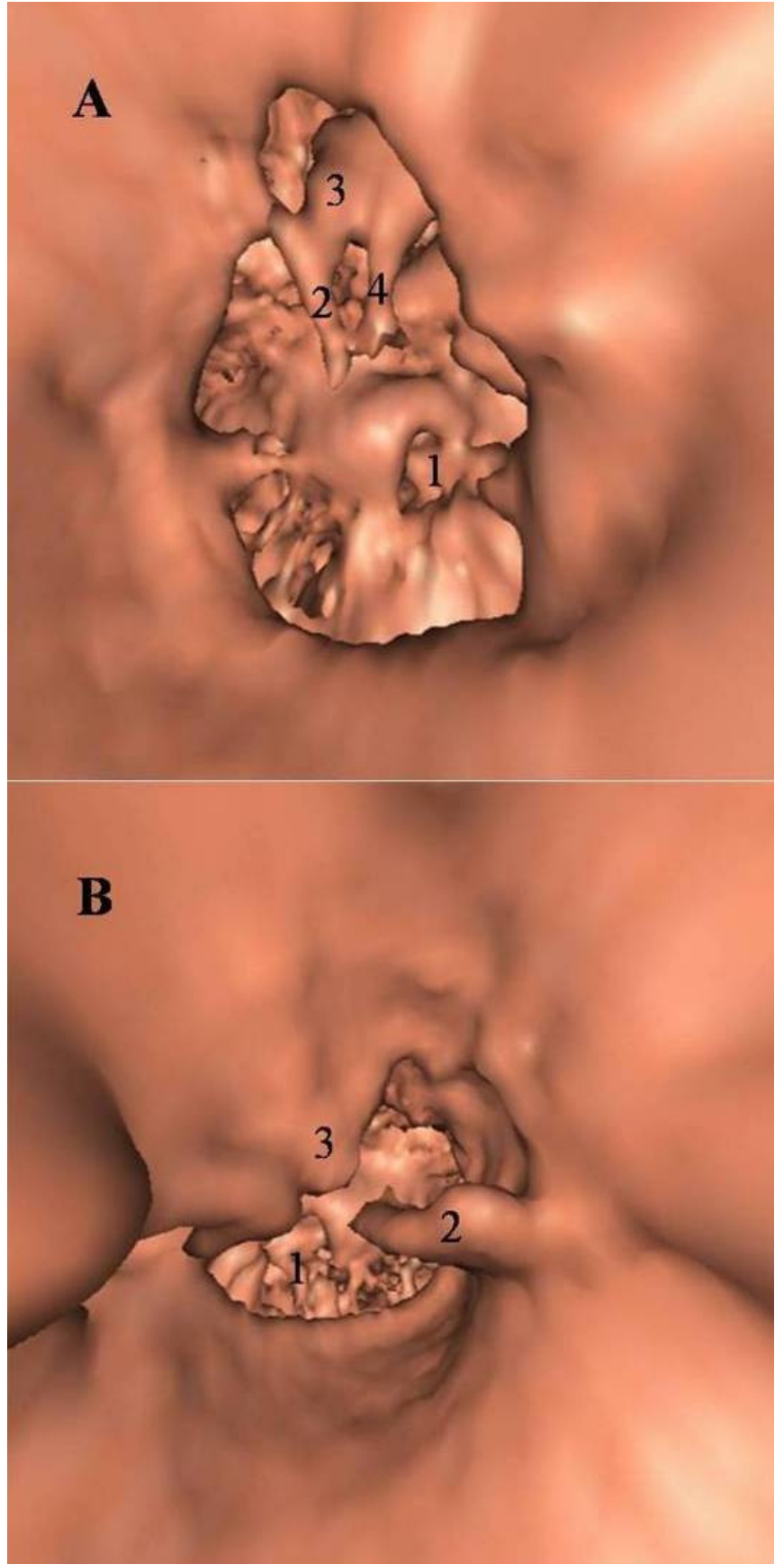

Fig. (2). VE of the left and right ears. (A) The normal left ear: 1Round window niche, 2-Handle of malleus, 3-Head of Malleus, 4Long process of incus. (B) The right ear: 1-Round window niche, 2Fractured and laterally and anteriorly dislocated handle of malleus, 3-bony ridge. The ossicular chain is not seen in the middle ear cavity.

not have provided adequate exposure of the ossicles because of the bony canal ridge caused by the fracture. Note that this was apparent only after the 3D reconstruction. This bony ridge would require drilling for a better exposure. Although, this entity is rare, based on our experience we suggest that VE should be part of the evaluation of various middle ear pathologies since it adds important information that cannot be easily extracted from the 2D scan. This added data does not necessitate any more imaging other than high resolution 


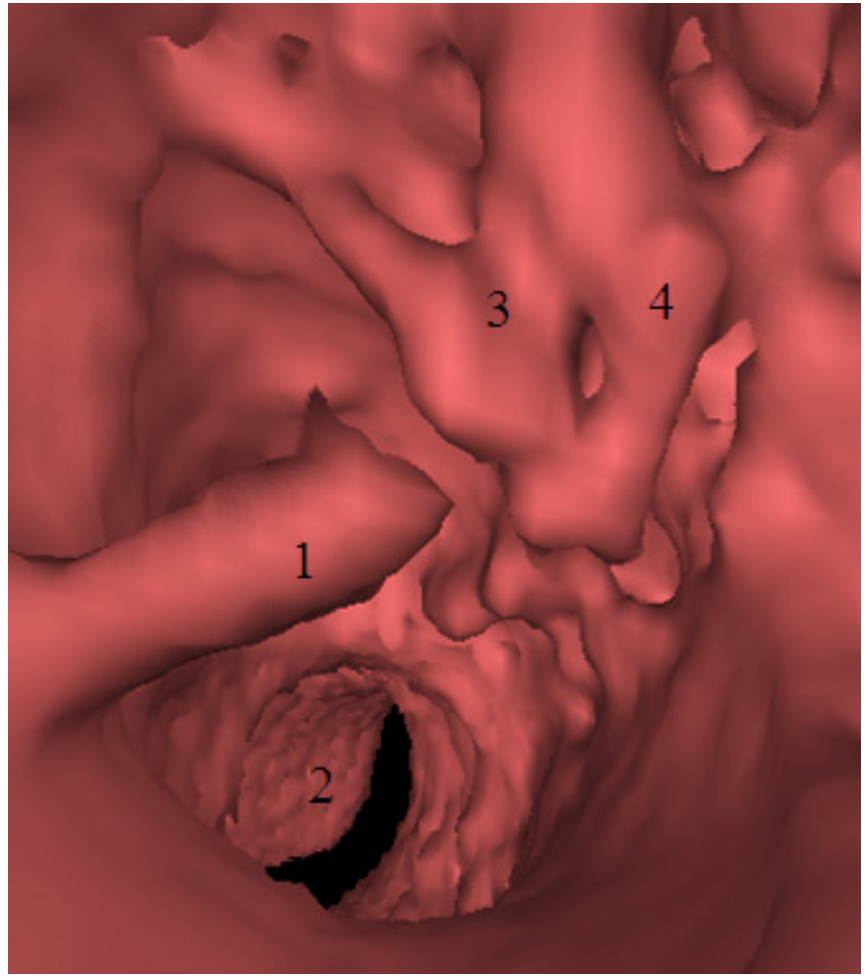

Fig. (3). VE of the right ear. A rotated view from the promontorium to the external ear canal.

1 - Fractured and dislocated handle of malleus, 2 - External acoustic meatus, 3 - Head of malleus, 4 - Incus.
CT that is preformed routinely for the evaluation of the middle ear. Slice width of $0.6 \mathrm{~mm}$ and slice increment of $0.3 \mathrm{~mm}$ that is used in routine temporal bone CT is a good base of $3 \mathrm{D} V E$ reconstruction image. The only necessary equipment is a powerful workstation commonly found today in many CT units and viewing software. This method can be facilitated by having at least one technician dedicated to 3D operations in the CT unit.

\section{REFERENCES}

[1] Iurato S, Quaranta A. Malleus-handle fracture: historical review and three new cases. Am J Otol 1999; 20: 19-25.

[2] Meriot P, Veillon F, Garcia JF, et al. CT appearances of ossicular injuries. Radiographics 1997; 17: 1445-54.

[3] Ayache D, Williams MT. Malleus handle fracture. Otol Neurotol 2003; 24: 519-20.

[4] Himi T, Sakata M, Shintani T, et al. Middle ear imaging using virtual endoscopy and its application in patients with ossicular chain anomaly. ORL J Otorhinolaryngol Relat Spec 2000; 62: 31620.

[5] Martin C, Michel F, Pouget JF, Veyret C, Bertholon P, Prades JM. Pathology of the ossicular chain: comparison between virtual endoscopy and 2D spiral CT-data. Otol Neurotol 2004; 25: 215-9.

[6] Rodt T, Bartling S, Schmidt AM, Weber BP, Lenarz T, Becker H. Virtual endoscopy of the middle ear: experimental and clinical results of a standardised approach using multi-slice helical computed tomography. Eur Radiol 2002; 12: 1684-92.

[7] Applebaum EL, Goldin AD. Surgical management of isolated malleus handle fractures. Laryngoscope 2000; 110: 171-3. 